\title{
Identification of high-risk plaque features in intracranial atherosclerosis: initial experience using a radiomic approach
}

\author{
Zhang Shi ${ }^{1} \cdot$ Chengcheng Zhu ${ }^{2} \cdot$ Andrew J. Degnan ${ }^{3} \cdot$ Xia Tian $^{1} \cdot$ Jing $\mathrm{Li}^{1} \cdot$ Luguang Chen $^{1} \cdot$ Xuefeng Zhang ${ }^{1}$. \\ Wenjia Peng ${ }^{1} \cdot$ Chao Chen ${ }^{1} \cdot$ Jianping Lu ${ }^{1} \cdot$ Tao Jiang $^{1} \cdot$ David Saloner $^{2} \cdot$ Qi Liu ${ }^{1} \mathbb{C}$
}

Received: 30 October 2017 / Revised: 15 February 2018 / Accepted: 20 February 2018 / Published online: 9 April 2018

(C) The Author(s) 2018

\begin{abstract}
Objectives To evaluate a quantitative radiomic approach based on high-resolution magnetic resonance imaging (HR-MRI) to differentiate acute/sub-acute symptomatic basilar artery plaque from asymptomatic plaque.

Methods Ninety-six patients with basilar artery stenosis underwent HR-MRI between January 2014 and December 2016. Patients were scanned with T1- and T2-weighted imaging, as well as T1 imaging following gadolinium-contrast injection (CE-T1). The stenosis value, plaque area/burden, lumen area, minimal luminal area (MLA), intraplaque haemorrhage (IPH), contrast enhancement ratio and 94 quantitative radiomic features were extracted and compared between acute/sub-acute and asymptomatic patients. Multi-variate logistic analysis and a random forest model were used to evaluate the diagnostic performance.

Results IPH, MLA and enhancement ratio were independently associated with acute/subacute symptoms. Radiomic features in $\mathrm{T} 1$ and CE-T1 images were associated with acute/subacute symptoms, but the features from T2 images were not. The combined IPH, MLA and enhancement ratio had an area under the curve (AUC) of 0.833 for identifying acute/sub-acute symptomatic plaques, and the combined T1 and CE-T1 radiomic approach had a significantly higher AUC of $0.936(p=0.01)$. Combining all features achieved an AUC of 0.974 and accuracy of $90.5 \%$.

Conclusions Radiomic analysis of plaque texture on HR-MRI accurately distinguished between acutely symptomatic and asymptomatic basilar plaques.

Key Points

- High-resolution magnetic resonance imaging can assess basilar artery atherosclerotic plaque.

- Radiomic features in T1 and CE-T1 images are associated with acute symptoms.

- Radiomic analysis can accurately distinguish between acute symptomatic and asymptomatic plaque.

- The highest accuracy may be achieved by combining radiomic and conventional features.
\end{abstract}

Keywords Intracranial arteriosclerosis $\cdot$ Magnetic resonance imaging $\cdot$ Stroke $\cdot$ Atherosclerotic plaques $\cdot$ Basilar artery

Zhang Shi and Chengcheng Zhu contributed equally to this work.

Electronic supplementary material The online version of this article (https://doi.org/10.1007/s00330-018-5395-1) contains supplementary material, which is available to authorized users.

Qi Liu

liuqimd@126.com

1 Department of Radiology, Changhai Hospital, Second Military Medical University, Shanghai 200433, China

2 Department of Radiology and Biomedical Imaging, UCSF, San Francisco, CA, USA

3 Department of Radiology, The Children's Hospital of Philadelphia, Philadelphia, PA, USA

\begin{tabular}{ll}
\multicolumn{2}{l}{ Abbreviations } \\
BA & Basilar artery \\
GLCM & Grey-level co-occurrence matrix \\
GLRLM & Grey-level run-length matrix \\
GLSZM & Grey-level size-zone matrix \\
HR-MRI & $\begin{array}{l}\text { High-resolution magnetic } \\
\text { resonance imaging }\end{array}$ \\
ICAD & Intracranial atherosclerotic disease \\
ICC & Intraclass coefficient \\
IPH & Intraplaque haemorrhage \\
MLA & Minimal luminal area \\
PB & Plaque burden
\end{tabular}




\section{Introduction}

Increasing evidence supports the presentation of intracranial atherosclerotic disease (ICAD) as a major source of ischaemic cerebrovascular events worldwide, particularly in Asian, African American and Hispanic populations [1, 2]. The massive global health burden of ICAD is only partially understood despite increasing awareness including recent population investigations that implicate ICAD in dementia [3, 4]. Given the high risk of stroke recurrence in symptomatic patients with ICAD, accurate risk assessment of intracranial plaque is essential in guiding clinical management [1].

The traditional assessment of ICAD is by evaluation of luminal narrowing based on angiographic methods including: digital subtraction angiography (DSA), computed tomographic angiography (CTA) and magnetic resonance angiography (MRA) [5]. Due to the phenomenon of positive remodelling, in which an artery compensates for plaque growth by expansion of the outer wall, leaving the luminal diameter relatively unaffected, these angiographic methods have underestimated ICAD plaque burden, and an autopsy study attributed up to $27 \%$ of fatal ischaemic strokes to intracranial plaque with mild to moderate (30-75\%) stenosis [6].

High-resolution magnetic resonance imaging (HR-MRI) of intracranial vessel walls facilitates the reliable, non-invasive characterisation of intracranial plaque, with clinical studies supporting its utility in ischaemic stroke risk estimation and ex vivo work validating its ability to classify plaque features [5, 7-13]. Posterior circulation plaques may be particularly underestimated by angiographic means because the basilar artery exhibits a propensity towards positive remodelling and the posterior circulation is predisposed to perforator occlusion [14-16]. Vertebrobasilar artery stenosis confers a significant risk of stroke, myocardial infarction and death [17]. Prior work supports the improved accuracy of HR-MRI over MRA in assessing basilar artery stenosis and its usefulness in guiding endovascular interventions [18-22].

Despite the favourable preliminary results, HR-MRI of intracranial plaque is complex and requires subjective assessment of plaque components. Advances in computing have enabled the development of automated, reproducible analysis methods, termed radiomics, to extract information from imaging studies to infer prognostic information for individual patients [23]. Radiomic approaches, such as texture analysis, show promise in multiple pathologies, including glioma, prostate cancer, hepatocellular carcinoma, and lung cancer, across different imaging modalities [24-27]. These radiomic approaches have not yet been applied to HR-MRI of ICAD.

This HR-MRI study of patients with ICAD including basilar artery plaque seeks to ascertain the ability of a radiomic approach including texture analysis to differentiate acute/subacute symptomatic plaque from asymptomatic plaque to serve as a framework for future prospective evaluations of basilar artery plaque risk.

\section{Materials and methods}

\section{Study population}

This study was approved by the Institutional Review Board of the Changhai Hospital (Shanghai, China), with all patients providing written informed consent. This study population was retrospectively selected from patients with intracranial stenosis who underwent intracranial HR-MRI between January 2014 and December 2016. All investigations were performed in accordance with the approved guidelines, including anonymisation of data during analysis. Patient inclusion criteria: (1) ischaemic stroke in basilar artery territory for the symptomatic group; (2) basilar artery stenosis on MRA or CTA; (3) $\geq 1$ atherosclerotic risk-factors, including hypertension, diabetes mellitus, hypercholesterolaemia or cigarette smoking. Exclusion criteria: (1) nonatherosclerotic intracranial arterial disease, including vasculitis, moyamoya disease, dissection, reversible cerebral vasoconstriction syndrome and intracranial dolichoectasia; (2) chronic ischaemic stroke/transient ischaemic attack (TIA) ( $>12$ weeks); (3) significant stenosis of the extracranial carotid arteries (> 30\%) as assessed on ultrasound; (4) ascending aortic arch atheroma; (5) suspected cardioembolic stroke; (6) known coagulopathy; (7) clinical contraindications to MRI, such as patients with pacemakers, certain metallic implants or severe claustrophobia.

\section{MRI acquisition}

Patients were scanned using a 3-T whole-body MRI scanner (Skyra; Siemens Healthcare, Erlangen, Germany) with a 20channel phased array head and neck coil. Patients were scanned using two dimensional high-resolution black blood T1- and T2- weighted fast-spin-echo sequences. T1 images were acquired both pre- and post-contrast (gadolinium). After an initial multi-plane localiser sequence, axial threedimensional (3D) time-of-flight (TOF) MR angiography was performed to identify the location of the basilar stenosis. Then HR-MRI scanning was performed in planes perpendicular to the longitudinal orientation of the artery. Scan parameters: both T1- and T2-weighted sequences were acquired with 12 $\times 2$-mm-thick slices; field of view $100 \mathrm{~mm} \times 100 \mathrm{~mm}$; matrix $256 \times 320$; in-plane resolution of $0.4 \mathrm{~mm} \times 0.3 \mathrm{~mm}$. T1 images had TR/TE $=581 \mathrm{~ms} / 18 \mathrm{~ms}$, echo train length $(\mathrm{ETL})=4$ and number of excitations $(\mathrm{NEX})=4$. T2 images had TR/TE $=2,890 \mathrm{~ms} / 46 \mathrm{~ms} ; \mathrm{ETL}=20 ; \mathrm{NEX}=3$. 


\section{Image analysis}

All images were analysed by four radiologists (two radiology residents each with 3 years' experience and two senior radiologists with 8 years' experience in intracranial vessel wall imaging using these dedicated sequences). Each detected plaque was independently classified as acute/sub-acute symptomatic if a lesion was present on conventional neuroimaging (T2 FLAIR and DWI showed infarct) after an acute ( $<4$ weeks) or sub-acute (4-12 weeks) ischaemic stroke/TIA with corresponding clinical symptoms. When multiple stenoses were present along the basilar artery, classification was based on the plaque at the location of maximal stenosis. A plaque was categorised as asymptomatic if detected in an asymptomatic patient and where conventional neuroimaging provided no evidence of infarct within the supplied vascular territory. Any disagreement was resolved by consensus.

Stenosis values were measured independently on HR-MRI images by one experienced radiologist (5 years' experience in neuroradiology) [28]. Presence of fresh intraplaque haemorrhage (IPH) was identified if the signal intensity was $>150 \%$ that of the nearby auricularis anterior muscles on pre-contrast T1-weighted images [29]. Lumen and outer wall boundaries were manually segmented on T2-weighted images at the slice with maximum plaque area using CMR Tools software (Cardiovascular Imaging Solutions, London, UK). Minimal luminal area (MLA) was computed based on the segmentation. MR signal intensity may vary within individuals due to factors such as coil positioning. Reproducibility of this area measurement method was previously reported to be excellent [30].

Gadolinium-based contrast agents were injected and postcontrast imaging was performed. The contrast enhancement ratio was measured at the slice of greatest enhancement, using adjacent grey matter (in a region of $\sim 15 \mathrm{~mm}^{2}$ at the hippocampus) to normalise the signal intensity. The contrast enhancement percentage was calculated as \{ (signal of plaque [post-contrast]/ signal of grey matter [post-contrast] ) / (signal of plaque [precontrast]/signal of grey matter[pre-contrast] $)-1\} \times 100 \%$. Plaque burden (PB) was measured at the site of maximal stenosis, and was defined as $(1-$ lumen area/outer area $) \times 100 \%$.

The radiomic features were analysed by an experienced reviewer (5 years' experience in vessel wall imaging) using dedicated software (3D-Slicer) [31]. To evaluate the reproducibility of the radiomic analysis, another reviewer (10 years' experience in vessel wall imaging) independently measured the radiomic features in a subset of patients $(n=40)$ who were randomly selected from the study population. Each reviewer segmented the plaque boundaries at the level of the largest plaque area on T1, T2 and CE-T1 images. For each sequence, 94 radiomic features - including intensity (maximal intensity, mean intensity, standard deviation of intensity, etc), shapebased features (area, length, etc.), and textures - were extracted and analysed according to a previous publication [32].
Textures include grey-level co-occurrence matrix (GLCM), grey-level run-length matrix (GLRLM) and grey-level sizezone matrix (GLSZM). The textural features were described patterns or the spatial distribution of voxel intensities, which were calculated respectively from grey level co-occurrence (GLCM) and grey-level run-length (GLRLM) texture matrices. Determining texture matrix representations requires the voxel intensity values within the volume of interest (VOI) to be discretised. This discretisation step not only reduces image noise but also normalises intensities across all patients, allowing for a direct comparison of all calculated textural features between patients. The detailed definition of these features can be found in the previous publication [32]. The reproducibility of radiomic feature quantification was calculated by comparing the results from the two reviewers.

\section{Statistical analysis}

All statistical analyses were performed using MATLAB (R2013a; The Mathworks, Natick, MA, USA) and SPSS24.0. The mean and standard deviation (SD) were recorded for continuous variables, and frequency and percentage were recorded for categorical variables. For each variable, a normality test was performed. Univariate analysis was first performed to assess the association between each variable and acute symptomatic status. As all variables obtained from the radiomic analysis exhibited normal distributions, $t$-tests were used for the comparison of continuous variables. Chi-squared tests were used for the categorical variables. Multivariate analysis was then performed which included the variables that had $p<0.10$ in the univariate tests. The odds ratios (ORs) with 95\% confidence intervals (CIs) were calculated by a logistic regression model with stepwise selection of variables. Additionally, $t$-tests were used to first select the features with $p<0.05$ in each sequence, and the features with significant differences and area under curve (AUC) values $>0.65$ were set as inputs for the random forest training features. To evaluate the diagnostic performance of radiomic features, supervised machine-learning methods using random forests [33] were applied to classify acute symptomatic from asymptomatic plaques.

A random forest is a meta-estimator that fits a number of decision-tree classifiers on various sub-samples of the dataset; ten classification trees with three layers were applied in this model; after each tree was voted the decision for classification result, the result was averaged to improve the predictive accuracy and control over-fitting. In random forests, each tree in the ensemble is built from a sample drawn with replacement from the training set. In addition, when splitting a node during the construction of the tree, the split that is picked is the best split among a random subset of the features.

The diagnostic performance was described using receiver operating characteristic (ROC) curves and AUC values. ROC 
curves were compared using the method developed by DeLong et al. [34] Reproducibility was evaluated by BlandAltman plots and intraclass coefficient (ICC) using a two-way random model with absolute measurements. A $p$ value $<0.05$ was considered statistically significant.

\section{Results}

\section{Patients}

A total of 174 patients met the inclusion criteria. Seventyeight patients were excluded due to intracranial aneurysm (n $=51)$, moyamoya disease $(n=13)$, vasculitis $(n=7)$, dissection $(n=5)$ and bad image quality $(n=2)$. As a result, 96 patients were included in the final analysis. The demographic data of the patients is shown in Table 1. There were 43 acute, 18 sub-acute and 35 asymptomatic patients.

\section{Traditional assessment of the intracranial atherosclerotic plaques}

Clinical and radiological characteristics of the intracranial atherosclerotic plaques are summarised in Table 2. Sample patient images are shown in Figs. 1 and 2. Univariate analysis showed that gender, smoking, IPH, MLA and enhancement ratio were associated with acute/sub-acute symptomatic plaques $(p<0.1)$. Multivariate logistic regression analysis showed that IPH [odds ratio $(\mathrm{OR})=17.803 ; 95 \% \mathrm{CI}$, 2.093151.472], MLA (OR $=1.515 ; 95 \% \mathrm{CI}, 1.123-2.043)$ and enhancement ratio $(\mathrm{OR}=71.979 ; 95 \% \mathrm{CI}, 3.840-1349.211)$ were independent predictors of acute/sub-acute symptoms, and the AUC of the ROC curves was $0.638,0.650$ and

Table 1 Patient demographic data

\begin{tabular}{ll}
\hline Characteristics & $n /$ total $(\%)$ \\
\hline Gender & \\
$\quad$ Male & $64(66.67)$ \\
Female & $32(33.33)$ \\
Age $^{\mathrm{a}}$ & $61.85 \pm 10.08$ \\
Diabetes mellitus & $34(35.05)$ \\
Hypertension & $78(80.41)$ \\
Hyperlipidaemia & $49(50.52)$ \\
Smoking & $27(27.84)$ \\
Stenosis (>50\%) & $60(61.86)$ \\
Clinical symptom & \\
Acute symptomatic & $43(44.79)$ \\
Sub-acute symptomatic & $18(18.75)$ \\
Asymptomatic & $35(36.46)$ \\
\hline
\end{tabular}

${ }^{\mathrm{a}}$ Mean $( \pm \mathrm{SD})$
0.717, respectively (Fig. 3). The AUC value was improved to 0.833 when combining IPH, MLA and enhancement ratio in the model, with an optimised sensitivity of $84.8 \%$, specificity of $71.0 \%$ and accuracy of $74.7 \%$. Details of the diagnostic performance data are listed in Table 4.

\section{Radiomic assessment of intracranial atherosclerotic plaques}

Samples of the radiomic features are shown in Fig. 2. Random forest analysis showed seven radiomic features in $\mathrm{T} 1$ images and three features in CE-T1 images that were independently associated with acute/sub-acute symptoms (Table 3), while none of the features in $\mathrm{T} 2$ images were statistically significant. Diagnostic performance data of radiomics are listed in Table 4. T1 radiomic features had an AUC value of 0.893 and CE-T1 radiomic features, 0.918 . When combining all the radiomic features from $\mathrm{T} 1$ and CE-T1 images, the AUC value improved to 0.936 , with an optimised sensitivity of $97.0 \%$ and specificity of $79.0 \%$, and accuracy of $83.2 \%$ (Fig. 3). Radiomic features had significantly higher AUC values than traditional features $(p=0.01)$.

When combining all radiomic features and IPH/MLA/enhancement ratio, the AUC value was improved to 0.974 ; however, it was not significantly higher than radiomic features alone $(p=0.275)$. The optimised sensitivity was $93.9 \%$, the specificity was $87.1 \%$ and the accuracy was $90.5 \%$.

\section{Reproducibility of traditional and radiomic measurements}

Bland-Altman plots of traditional and radiomic measurements and the ICC values are shown in Supplementary Figs. 1 and 2. The ICCs of the traditional features for the two reviewers in measuring the degree of stenosis, PB, MLA and enhancement ratio were $0.782,0.778,0.875,0.792$ respectively (average ICC, 0.806). The Cohen's kappa for the two reviewers in determining IPH was 0.811 .

The average ICCs for the two reviewers in measuring the ten independent radiomic features were 0.836 (range, 0.798 0.881 ) for $\mathrm{T} 1$ images and 0.837 (range, $0.806-0.858$ ) for CE$\mathrm{T} 1$ images.

\section{Discussion}

Quantitative radiomic analysis accurately differentiated symptomatic from asymptomatic basilar artery atherosclerotic plaques on HR-MRI, outperforming conventional imaging variables and clinical risk factors. As there is now increasing recognition that ICAD is more common than previously thought, with an estimated prevalence of ICAD in the United States of 34\% [10], it is apparent that characterisation 
Table 2 Clinical and radiological features of the intracranial atherosclerotic plaques

Intracranial atherosclerosis

Multivariate odds ratio $(95 \% \mathrm{CI})^{\mathrm{b}}$

\begin{tabular}{lll}
\hline $\begin{array}{l}\text { Acute/sub-acute } \\
\text { symptomatic }\end{array}$ & Asymptomatic
\end{tabular}

Gender

Male
Female

Age

Diabetes mellitus $^{\mathrm{c}}$

Hypertension $^{\mathrm{d}}$

Hyperlipidaemia $^{\mathrm{d}}$

Smoking

IPH

Plaque burden (\%)

MLA ( $\left.\mathrm{mm}^{3}\right)$

Degree of stenosis (\%)

Enhancement ratio (\%) symptomatic

$\begin{array}{llr}46 & 18 & 0.023 \\ 15 & 17 & \\ 61.68 \pm 10.75 & 62.14 \pm 8.92 & 0.828 \\ 23 & 11 & 0.716 \\ 48 & 30 & 0.323 \\ 23 & 11 & 0.574 \\ 23 & 4 & 0.013 \\ 19 & 1 & 0.003 \\ 83.24 \pm 9.71 & 85.04 \pm 7.51 & 0.345 \\ 3.78 \pm 2.80 & 2.39 \pm 1.46 & 0.008 \\ 53.69 \pm 15.19 & 53.76 \pm 16.73 & 0.984 \\ 24.20 \pm 29.46 & 3.38 \pm 21.91 & <0.001\end{array}$

0.023

$\begin{array}{ll}0.828 & \\ 0.716 & \\ 0.323 & \\ 0.574 & \\ 0.013 & \\ 0.003 & 17.803(2.093,151.472) p=0.008 \\ 0.345 & \\ 0.008 & 71.979(3.840,1349.211) p=0.004 \\ 0.984 & \\ 0.001 & \end{array}$

IPH intraplaque haemorrhage, MLA minimum luminal area

${ }^{\text {a }}$ Two independent-samples $t$-test for continuous variables

${ }^{\mathrm{b}}$ Results from multivariate analysis

${ }^{\mathrm{c}}$ Diabetes mellitus: two fasting glucose measurements above $126 \mathrm{mg} / \mathrm{dl}(7.0 \mathrm{mmol} / \mathrm{l})$ or glycated haemoglobin $(\mathrm{HbA} 1 \mathrm{C}) \geq 48 \mathrm{mmol} / \mathrm{mol}$

${ }^{\mathrm{d}}$ Hypertension: a systolic or a diastolic blood pressure measurement consistently higher than an accepted normal value (this is above $139 \mathrm{mmHg}$ systolic, $89 \mathrm{mmHg}$ diastolic)

${ }^{\mathrm{e}}$ Hyperlipidemia: abnormally elevated levels of any or all lipids or lipoproteins in the blood;LDL-C $>130 \mathrm{mg} / \mathrm{dl}$, HDL-C $<40 \mathrm{mg} / \mathrm{dl}$, TG $>150 \mathrm{mg} / \mathrm{dl}$, TC $>200 \mathrm{mg} / \mathrm{dl}$

of intracranial plaque must go beyond assessing the presence of plaque or the degree of stenosis to accurately determine the risk of future cerebrovascular event. In this study, radiomic analysis accurately assigned symptom status to basilar artery plaque with an excellent AUC of 0.936, while clinical imaging features alone had a moderate AUC of 0.833 . This retrospective study suggests radiomic analysis could substantially improve assessment of acute symptomatic versus chronic/ asymptomatic basilar intracranial atherosclerosis.

Qualitative features of intracranial plaque such as plaque enhancement, suggesting active inflammation, or $\mathrm{T}_{1}$-weighted hyperintense signal, indicating intraplaque haemorrhage, have been associated with symptomatic, culprit plaque $[11,35]$. In addition, quantitative plaque features such as stenosis, plaque area and enhancement ratio may also be helpful. However, both qualitative and quantitative imaging features are limited by variable observer expertise, limited reproducibility and heterogeneous imaging protocols. A key advantage of the radiomic method is that it can provide reader-independent findings [23]. These radiomic features cannot be readily discerned by a human observer and may substantially add to the diagnostic confidence and accuracy of a study in ways that a human interpreter is not capable of appreciating. In our analysis, the highest AUC (0.974) was found using radiomic basilar artery plaque features in conjunction with an assessment of MLA, the presence of intraplaque haemorrhage and gadolinium enhancement.

Compared with conventional methods, radiomic analysis is more quantitative and may detect "agnostic" features that the radiologist cannot appreciate, such as uniformity or randomness (entropy) of the intensities on the image. Radiomics provides richer information about intensity, shape, size or volume, and texture of the plaque/tumour. On the other hand, conventional measurements only provide limited information about image features. For example, IPH has been studied as a potential marker of high-risk intracranial plaque, but previous studies commonly used a binary categorisation (present or not) [29]. The quantitative signal intensity, volume/shape and the complicated distribution of IPH (focal or diffuse, close to the lumen or close to the wall) have rarely been studied, because routine image analysis by clinical radiologists does not yield this level of assessment. A quantitative radiomics matrix contains detailed information.

Because of these advantages, radiomics has shown great prognostic value. For instance, a study by Aerts et al. [32] showed that a prognostic radiomic signature, capturing intratumour heterogeneity, was associated with underlying gene-expression patterns in lung and head-and-neck cancer patients. These data suggest that radiomics identifies prognostic phenotypes existing in both lung and head-and-neck 
Fig. 1 MRI images showing BA atherosclerotic plaque in a symptomatic patient. TOF-MRA (a) demonstrates stenosis, and DWI (b) shows the acute infarcts which are scattered and patchy in distribution within the left cerebellum. T2-weighted, T1weighted and CE-T1-weighted images (from left to right) in the middle slice of the BA plaque are shown in $\mathbf{c}$ and $\mathbf{d}$
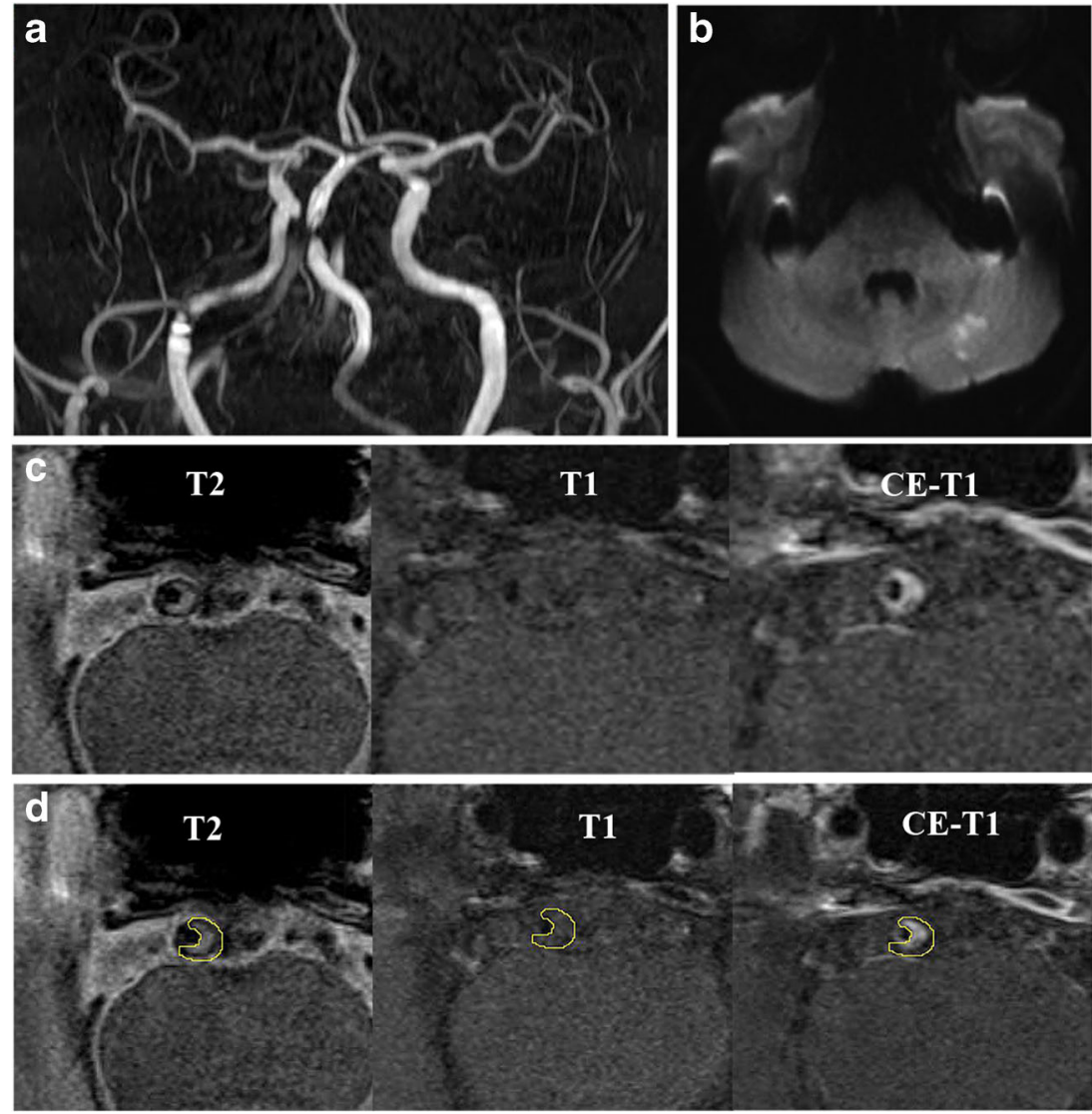

cancer. In a study of 282 patients with early-stage non-small cell lung cancer, radiomics showed additional value other than clinical-pathological risk factors for estimation of disease-free survival time [36].

We found the independent radiomics features from $\mathrm{T} 1$ and CE-T1 images were different. This is because the signal characteristics in pre- and post-contrast T1-weighted images reflect different pathophysiological features of plaque. For example, the high signal on pre-contrast T1-weighted images is possibly intraplaque haemorrhage (IPH), and the low signal is thought to represent the lipid core (as described in an ex vivo study [13]); on the other hand, the high signal on post-contrast T1-weighted images is attributed to the contrast uptake by fibrous cap or possible vasa vasorum.

In our study, the random forest method has been chosen. As a result of this randomness, the bias of the forest usually slightly increases (with respect to the bias of a single nonrandom tree) but, due to averaging, its variance also decreases, usually more than compensating for this increase in bias, yielding a better overall model. This procedure leads to better model performance because it decreases the variance of the model, without increasing the bias. This means that while the predictions of a single tree are highly sensitive to noise in its training set, the average of many trees is not, as long as the trees are not correlated. Simply training many trees on a single training set would give strongly correlated trees (or even the same tree many times, if the training algorithm is deterministic); bootstrap sampling is a way of de-correlating the trees by showing them different training sets.

To our knowledge, this study is the first investigation of ICAD with HR-MRI using a radiomic analysis. An ultrasound study of extra-cranial carotid artery atherosclerotic plaque applied texture feature analysis to classify plaque echogenicity with success [37]. Those authors achieved an accuracy of $88 \%$ in identifying anechoic, intermediate, and hyperechoic plaques with an AUC of 0.918 for the anechoic plaque, which is thought to confer an increased risk of cardiovascular and cerebrovascular events [37]. Another group used principal component analysis of carotid plaque texture and echogenicity to determine variables associated with plaque instability on histology that were independent of stenosis [38]. Texture analysis methods have also been applied to carotid ultrasound elastography using HR-MRI as a reference to improve plaque vulnerability assessment [39]. These ultrasound-based studies of extracranial atherosclerosis support the use of radiomic analysis to reveal quantitative variables within images to improve risk estimates associated with atherosclerotic plaque beyond plaque size or qualitative features. This study similarly extends radiomic analysis of intracranial plaque by classifying intracranial basilar artery plaque into acute/sub-acute 


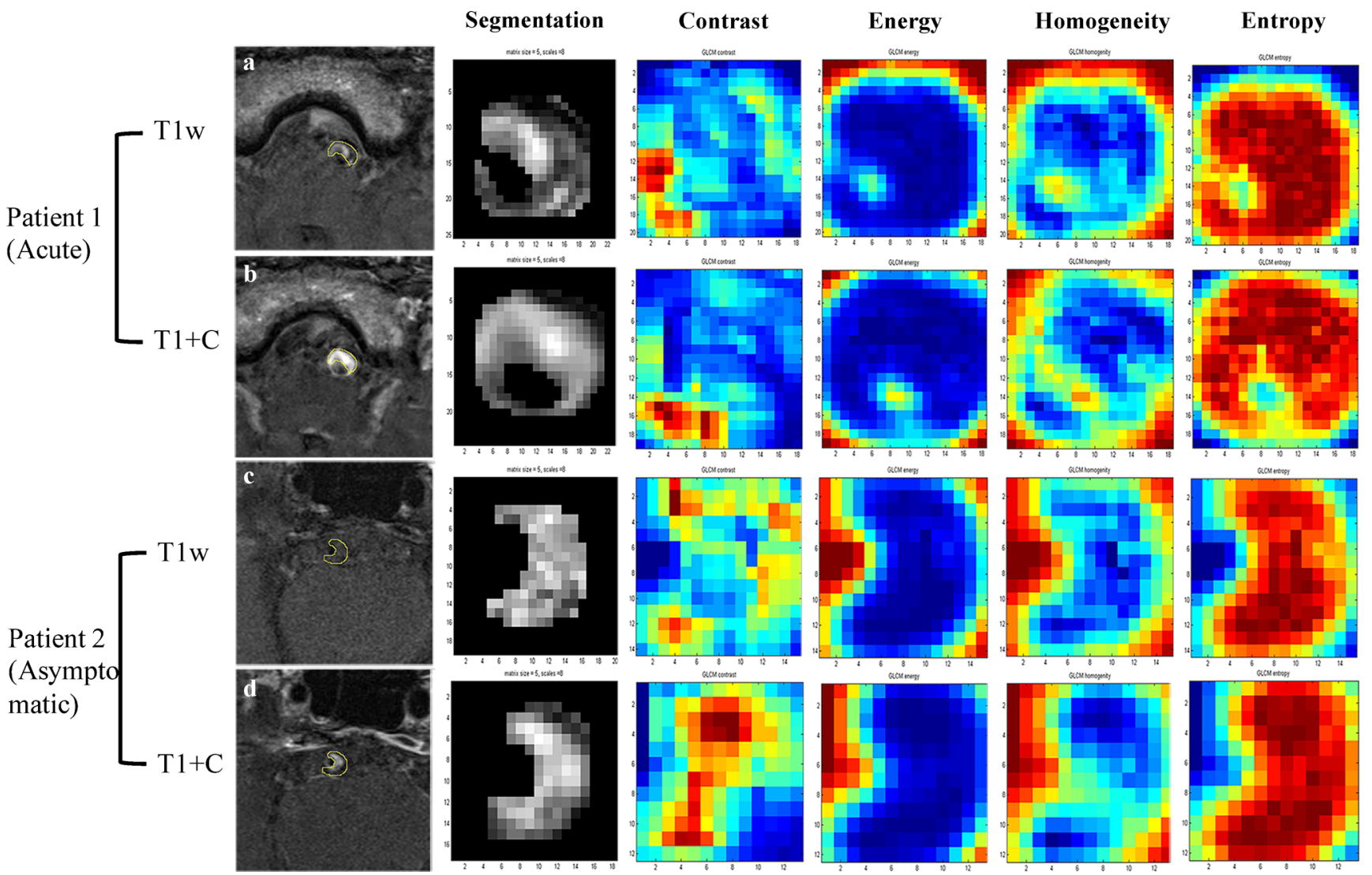

Fig. 2 Radiomics analysis in two sample patients. Patient 1 is a 63-yearold man with acute stroke on the stem. Patient 2 is a 57 -year-old man free of symptoms. T1-weighted images are shown in a and $\mathbf{c}$, and CE-T1

images are shown in $\mathbf{b}$ and $\mathbf{d}$. Four representative GLCM radiomics features from 94 features are shown (contrast, energy, homogeneity, entropy)

symptomatic versus asymptomatic based on quantitative information extracted from HR-MRI.

Measurements that include plaque burden and minimum luminal area as determined on HR-MRI have substantially

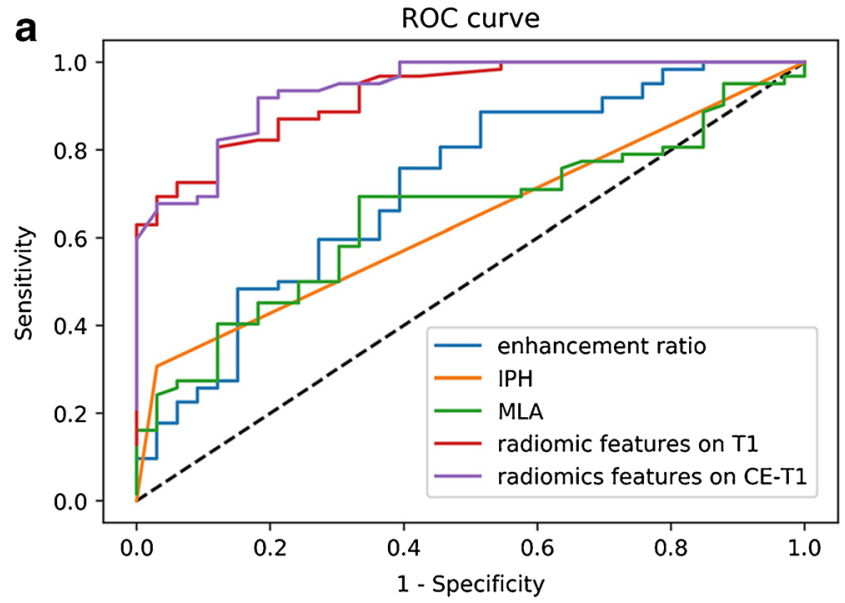

Fig. 3 ROC curves to differentiate acute/sub-acute symptomatic and asymptomatic plaques. The curves on the left (a) show the diagnostic performance of each independent parameter. The curves on the right (b) shows diagnostic performance of the combined traditional/radiomics improved the assessment of culprit plaques compared with degree of stenosis alone in a study of middle cerebral artery atherosclerosis [40]. However, the diagnostic performance of these variables was suboptimal, with the combination of plaque

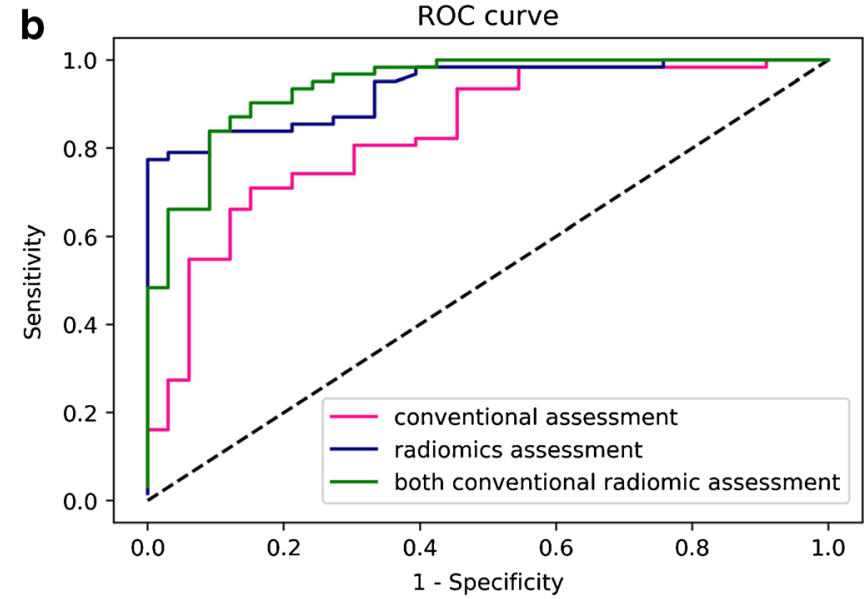

model and a model combination of all features. Radiomic features had significantly higher AUC values compared with traditional features $(p=$ $0.01)$ 
Table 3 Independent radiomics features on $\mathrm{T} 1$ and CE-T1 images

\begin{tabular}{lcrr}
\hline & Acute/sub-acute symptomatic & Asymptomatic & $p$ value \\
\hline Features on T1 images & & & \\
shape_Maximum3DDiameter & $5.16 \pm 1.41$ & $4.25 \pm 0.95$ & $<0.001$ \\
shape_Maximum2DDiameterSlice & $5.21 \pm 1.42$ & $4.27 \pm 0.98$ & $<0.001$ \\
shape_Volume & $40.16 \pm 24.01$ & $27.41 \pm 14.63$ & 0.005 \\
shape_SurfaceArea & $71.83 \pm 29.23$ & $53.43 \pm 19.58$ & 0.001 \\
shape_Maximum2DDiameterColumn & $4.40 \pm 1.41$ & $3.61 \pm 0.91$ & 0.004 \\
glcm_Entropy & $5.78 \pm 0.75$ & $5.36 \pm 0.73$ & 0.011 \\
glrlm_RunLengthNonUniformity & $102.89 \pm 62.18$ & $37.03 \pm 16.46$ & 0.006 \\
Features on CE-T1 images & & & \\
firstorder_Uniformity & $0.07 \pm 0.03$ & $0.09 \pm 0.02$ & 0.005 \\
glcm_MaximumProbability & $0.04 \pm 0.02$ & $0.06 \pm 0.05$ & 0.004 \\
glcm_Entropy & $6.01 \pm 0.84$ & $5.43 \pm 1.21$ & 0.007 \\
\hline
\end{tabular}

Definitions of the radiomics features can be found in the reference: https://www.ncbi.nlm.nih.gov/pubmed/ 24892406 burden, minimum luminal area and stenosis together resulting in an accuracy of $71.5 \%$ [40], which is comparable to the accuracy reported in our study when using enhancement ratio, MLA and IPH (74.7\%). Radiomic analysis has the benefit of identifying quantitative variables within imaging data to improve accuracy (83.2\% as shown in this study) and diagnostic confidence beyond conventional measurements. The favourable accuracy values in this study over those previously reported by conventional HR-MRI support the use of radiomic analysis to improve identification of acute symptomatic plaque.

It is hoped that radiomic approaches applied to HR-MRI of the basilar artery will result in improved accuracy of stroke risk assessment, which is especially important given the highrisk nature of symptomatic basilar artery ICAD [17]. This risk assessment could then be used to initiate more effective secondary prevention strategies by more appropriately assigning high-risk patients with posterior circulation ischaemic events due to non-stenotic plaque to more aggressive dual antiplatelet therapy. Before this radiomic plaque information can be applied more broadly, prospective treatment studies are needed to investigate the role of aggressive medical management in patients with non-stenotic, high-risk plaque as current treatment recommendations are based on prior investigations showing the benefit of dual antiplatelet therapy such as SAMMPRIS that was restricted to patients with high-grade stenosis of 70-99\% occlusion [41, 42]. Others have suggested a possible role for endovascular therapy of selected patients with vertebrobasilar stenosis, and basilar artery plaque radiomic assessment may assist in risk assessment and treatment decision-making. However, more data are needed regarding the role endovascular therapy in these conditions [43].

\section{Limitations}

Although this study provided significant results, the sample size used to train the texture analysis of plaque was relatively small $(n=96)$. Future work would benefit from training with a larger data set. In addition, our analysis was performed using two-dimensional (2D) imaging data. It is possible that the use of 3D imaging acquisitions could better characterise plaque features for improved radiomic analysis. Also, reliance on a 2D TOF sequence to initially identify plaque may have underestimated the presence of non-stenotic atherosclerotic plaque. Three-dimensional imaging methods (up to $0.5 \mathrm{~mm}$ isotropic) [44] can reduce the partial volume effect of 2D acquisition and allow the detection of non-stenotic basilar artery or PCA plaques. However, the long scan time and wider point spread function induced by long echo trains must also be considered $[45,46]$. Another potential improvement is the use of automated segmentation. Manual segmentation of the small
Table 4 The diagnostic accuracy findings

\begin{tabular}{llllllc}
\hline & DA & AUC & sensitivity & specificity & LR+ & 1/LR- \\
\hline Traditional assessment & 0.747 & 0.833 & 0.848 & 0.71 & 2.926 & 4.686 \\
Radiomics (T1-weighted) & 0.8 & 0.893 & 0.909 & 0.726 & 3.318 & 7.986 \\
Radiomics (CE-T1) & 0.819 & 0.918 & 0.909 & 0.823 & 5.136 & 9.053 \\
All of radiomics & 0.832 & 0.936 & 0.97 & 0.79 & 4.619 & 26.33 \\
Traditional \& radiomics & 0.905 & 0.974 & 0.939 & 0.871 & 7.279 & 14.28 \\
\hline
\end{tabular}

$D A$ diagnostic accuracy, $A U C$ area under the curve, $L R+$ positive likelihood ratio, $L R$ - negative likelihood ratio 
intracranial plaque is still a challenging task. Automatic segmentation would reduce inter-observer variability and improve the feasibility of applying these methods to larger datasets.

This retrospective study was not able to ascertain the ability of radiomic analysis to predict the risk of future stroke risk, which is of paramount importance. Future prospective studies associating plaque features with long-term stroke risk through radiomic techniques could substantially shift the imaging paradigm from simple assessment of stenosis and subjective plaque components to a rigorous evaluation of objective and quantifiable plaque characteristics.

As a nascent field, radiomic methods may require additional refinement to improve accuracy. Diagnostic accuracy and predictive power are dependent on the quality of the data set utilised to train radiomic algorithms [23]. Leveraging quantitative imaging data with large population studies could provide actionable information to generate robust automated assessments of stroke risk based on vessel wall MRI of ICAD.

\section{Conclusions}

Radiomic analysis of basilar artery plaque texture on HR-MRI accurately distinguished between acute/sub-acute symptomatic and asymptomatic plaque. Radiomic features alone successfully predicted plaque symptom status over clinical imaging features in this retrospective study. Prospective studies are needed to further ascertain the ability of radiomic analysis of intracranial plaque HR-MRI to predict future stroke risk.

Funding This work was supported in part by the National Natural Science Foundation of China (No. 81670396, No. 31470910, No. 81701689, No. 31600755), NIH grant K99HL136883, the12th Five Year Plan medical key project PLA of China (BWS12J026), the Emerging Frontier Technology Joint Research Program of Shanghai Municipal Hospital of China (SHDC12013110), Special Program of Military Medicine of the Second Military Medical University (2011JS18), and Changhai Hospital 1255 Scientific Innovation Funds (CH125541000) for the financial support.

\section{Compliance with ethical standards}

Guarantor The scientific guarantor of this publication is Qi Liu.

Conflict of interest The authors of this manuscript declare no relationships with any companies, whose products or services may be related to the subject matter of the article.

Statistics and biometry Qi Liu kindly provided statistical advice for this manuscript.

No complex statistical methods were necessary for this paper.

Informed consent Written informed consent was obtained from all subjects (patients) in this study.

Ethical approval Institutional Review Board approval was obtained.

\section{Methodology}

- retrospective

- diagnostic or prognostic study

- performed at one institution

Open Access This article is distributed under the terms of the Creative Commons Attribution 4.0 International License (http:// creativecommons.org/licenses/by/4.0/), which permits unrestricted use, distribution, and reproduction in any medium, provided you give appropriate credit to the original author(s) and the source, provide a link to the Creative Commons license, and indicate if changes were made.

\section{References}

1. Jeng JS, Tang SC, Liu HM (2010) Epidemiology, diagnosis and management of intracranial atherosclerotic disease. Expert Rev Cardiovasc Ther 8:1423-1432

2. Wong LK (2006) Global burden of intracranial atherosclerosis. Int J Stroke 1:158-159

3. Suri MF, Qiao Y, Ma X et al (2016) Prevalence of intracranial atherosclerotic stenosis using high-resolution magnetic resonance angiography in the general population: The Atherosclerosis Risk in Communities Study. Stroke 47:1187-1193

4. Dearborn JL, Zhang Y, Qiao Y et al (2017) Intracranial atherosclerosis and dementia: The Atherosclerosis Risk in Communities (ARIC) Study. Neurology 88:1556-1563

5. Mandell DM, Mossa-Basha M, Qiao Y et al (2017) Intracranial Vessel Wall MRI: Principles and Expert Consensus Recommendations of the American Society of Neuroradiology. AJNR Am J Neuroradiol 38: 218-229

6. Mazighi M, Labreuche J, Gongora-Rivera F, Duyckaerts C, Hauw JJ, Amarenco P (2008) Autopsy prevalence of intracranial atherosclerosis in patients with fatal stroke. Stroke 39:1142-1147

7. Swartz RH, Bhuta SS, Farb RI et al (2009) Intracranial arterial wall imaging using high-resolution 3-tesla contrast-enhanced MRI. Neurology 72:627-634

8. Degnan AJ, Gallagher G, Teng Z, Lu J, Liu Q, Gillard JH (2012) MR angiography and imaging for the evaluation of middle cerebral artery atherosclerotic disease. AJNR Am J Neuroradiol 33:1427-1435

9. van der Kolk AG, Zwanenburg JJ, Brundel M et al (2011) Intracranial vessel wall imaging at 7.0-T MRI. Stroke 42:2478-2484

10. Qiao Y, Guallar E, Suri FK et al (2016) MR imaging measures of intracranial atherosclerosis in a population-based study. Radiology 280:860-868

11. Qiao Y, Zeiler SR, Mirbagheri S et al (2014) Intracranial plaque enhancement in patients with cerebrovascular events on highspatial-resolution MR images. Radiology 271:534-542

12. Jiang Y, Zhu C, Peng W et al (2016) Ex-vivo imaging and plaque type classification of intracranial atherosclerotic plaque using high resolution MRI. Atherosclerosis 249:10-16

13. Yang WJ, Chen XY, Zhao HL et al (2016) Postmortem study of validation of low signal on fat-suppressed T1-weighted magnetic resonance imaging as marker of lipid core in middle cerebral artery atherosclerosis. Stroke 47:2299-2304

14. Qiao Y, Anwar Z, Intrapiromkul J et al (2016) Patterns and implications of intracranial arterial remodeling in stroke patients. Stroke 47:434-440

15. Lopez-Cancio E, Matheus MG, Romano JG et al (2014) Infarct patterns, collaterals and likely causative mechanisms of stroke in symptomatic intracranial atherosclerosis. Cerebrovasc Dis 37:417-422

16. Ma N, Jiang WJ, Lou X et al (2010) Arterial remodeling of advanced basilar atherosclerosis: a 3-tesla MRI study. Neurology 75: 253-258 
17. The Warfarin-Aspirin Symptomatic Intracranial Disease (WASID) Study Group (1998) Prognosis of patients with symptomatic vertebral or basilar artery stenosis. Stroke 29:1389-1392

18. Klein IF, Lavallee PC, Schouman-Claeys E, Amarenco P (2005) High-resolution MRI identifies basilar artery plaques in paramedian pontine infarct. Neurology 64:551-552

19. Klein IF, Lavallee PC, Mazighi M, Schouman-Claeys E, Labreuche J, Amarenco P (2010) Basilar artery atherosclerotic plaques in paramedian and lacunar pontine infarctions: a high-resolution MRI study. Stroke 41:1405-1409

20. Kim YS, Lim SH, Oh KW et al (2012) The advantage of highresolution MRI in evaluating basilar plaques: a comparison study with MRA. Atherosclerosis 224:411-416

21. Jiang WJ, Yu W, Ma N, Du B, Lou X, Rasmussen PA (2011) High resolution MRI guided endovascular intervention of basilar artery disease. J Neurointerv Surg 3:375-378

22. Huang B, Yang WQ, Liu XT, Liu HJ, Li PJ, Lu HK (2013) Basilar artery atherosclerotic plaques distribution in symptomatic patients: a 3.0T high-resolution MRI study. Eur J Radiol 82:e199-e203

23. Gillies RJ, Kinahan PE, Hricak H (2016) Radiomics: images are more than pictures, they are data. Radiology 278:563-577

24. Rose CJ, Mills SJ, O'Connor JP et al (2009) Quantifying spatial heterogeneity in dynamic contrast-enhanced MRI parameter maps. Magn Reson Med 62:488-499

25. Wibmer A, Hricak H, Gondo T et al (2015) Haralick texture analysis of prostate MRI: utility for differentiating non-cancerous prostate from prostate cancer and differentiating prostate cancers with different Gleason scores. Eur Radiol 25:2840-2850

26. Segal E, Sirlin CB, Ooi C et al (2007) Decoding global gene expression programs in liver cancer by noninvasive imaging. Nat Biotechnol 25:675-680

27. Coroller TP, Grossmann P, Hou Y et al (2015) CT-based radiomic signature predicts distant metastasis in lung adenocarcinoma. Radiother Oncol 114:345-350

28. Liu Q, Huang J, Degnan AJ et al (2013) Comparison of highresolution MRI with CT angiography and digital subtraction angiography for the evaluation of middle cerebral artery atherosclerotic steno-occlusive disease. Int J Card Imaging 29:1491-1498

29. Yu JH, Kwak HS, Chung GH, Hwang SB, Park MS, Park SH (2015) Association of intraplaque hemorrhage and acute infarction in patients with basilar artery plaque. Stroke 46:2768-2772

30. Zhang X, Zhu C, Peng W et al (2015) Scan-rescan reproducibility of high resolution magnetic resonance imaging of atherosclerotic plaque in the middle cerebral artery. PLoS One 10:e0134913

31. Schlett CL, Maurovich-Horvat P, Ferencik M et al (2013) Histogram analysis of lipid-core plaques in coronary computed tomographic angiography: ex vivo validation against histology. Investig Radiol 48:646-653
32. Aerts HJ, Velazquez ER, Leijenaar RT et al (2014) Decoding tumour phenotype by noninvasive imaging using a quantitative radiomics approach. Nat Commun 5:4006

33. Shi T, Seligson D, Belldegrun AS, Palotie A, Horvath S (2005) Tumor classification by tissue microarray profiling: random forest clustering applied to renal cell carcinoma. Mod Pathol 18:547-557

34. DeLong ER, DeLong DM, Clarke-Pearson DL (1988) Comparing the areas under two or more correlated receiver operating characteristic curves: a nonparametric approach. Biometrics 44:837-845

35. Xu WH, Li ML, Gao S et al (2012) Middle cerebral artery intraplaque hemorrhage: prevalence and clinical relevance. Ann Neurol 71:195-198

36. Huang Y, Liu Z, He L et al (2016) Radiomics signature: a potential biomarker for the prediction of disease-free survival in early-stage (I or II) non-small cell lung cancer. Radiology 281:947-957

37. Huang X, Zhang Y, Qian M et al (2016) Classification of carotid plaque echogenicity by combining texture features and morphologic characteristics. J Ultrasound Med 35:2253-2261

38. Doonan RJ, Gorgui J, Veinot JP et al (2016) Plaque echodensity and textural features are associated with histologic carotid plaque instability. J Vasc Surg 64:671 677.e678

39. Huang C, Pan X, He Q et al (2016) Ultrasound-based carotid elastography for detection of vulnerable atherosclerotic plaques validated by magnetic resonance imaging. Ultrasound Med Biol 42:365-377

40. Teng Z, Peng W, Zhan Q et al (2016) An assessment on the incremental value of high-resolution magnetic resonance imaging to identify culprit plaques in atherosclerotic disease of the middle cerebral artery. Eur Radiol 26:2206-2214

41. Chimowitz MI, Lynn MJ, Derdeyn CP et al (2011) Stenting versus aggressive medical therapy for intracranial arterial stenosis. $\mathrm{N}$ Engl J Med 365:993-1003

42. Holmstedt CA, Turan TN, Chimowitz MI (2013) Atherosclerotic intracranial arterial stenosis: risk factors, diagnosis, and treatment. Lancet Neurol 12:1106-1114

43. Hussain M, Datta N, Cheng Z et al (2017) Spanning from the West to East: an updated review on endovascular treatment of intracranial atherosclerotic disease. Aging Dis 8:196-202

44. Qiao Y, Steinman DA, Qin Q et al (2011) Intracranial arterial wall imaging using three-dimensional high isotropic resolution black blood MRI at 3.0 Tesla. J Magn Reson Imaging 34:22-30

45. Chen Z, Liu AF, Chen $\mathrm{H}$ et al (2016) Evaluation of basilar artery atherosclerotic plaque distribution by $3 \mathrm{D}$ MR vessel wall imaging. J Magn Reson Imaging 44:1592-1599

46. Zhu C, Haraldsson H, Tian B et al (2016) High resolution imaging of the intracranial vessel wall at 3 and $7 \mathrm{~T}$ using 3D fast spin echo MRI. MAGMA 29:559-570 